\title{
Pour une autre lecture de la guerre de Gaza
}

\author{
Jean-François Legrain
}

\section{OpenEdition \\ Journals}

\section{Édition électronique}

URL : http://journals.openedition.org/echogeo/10901

DOI : $10.4000 /$ echogeo. 10901

ISSN : 1963-1197

\section{Éditeur}

Pôle de recherche pour l'organisation et la diffusion de l'information géographique (CNRS UMR 8586)

\section{Référence électronique}

Jean-François Legrain, « Pour une autre lecture de la guerre de Gaza », EchoGéo [En ligne], Sur le Vif, mis en ligne le 13 février 2009, consulté le 19 avril 2019. URL : http://journals.openedition.org/ echogeo/10901 ; DOI : 10.4000/echogeo.10901

Ce document a été généré automatiquement le 19 avril 2019.

\section{(c) (i) (9)}

EchoGéo est mis à disposition selon les termes de la licence Creative Commons Attribution - Pas d'Utilisation Commerciale - Pas de Modification 4.0 International 


\title{
Pour une autre lecture de la guerre de Gaza
}

\author{
Jean-François Legrain
}

1 Alors que l'armée israélienne s'est retirée de la bande de Gaza au terme d'une offensive qui aura duré plus de trois semaines (27 décembre 2008-21 janvier 2009) et fait quelque 1330 morts et 5450 blessés côté palestinien pour 10 soldats et 3 civils tués côté israélien, il convient de revenir tant sur les justifications de son déclenchement avancées par le gouvernement israélien que sur l'approche de son bilan menée en termes de victoire palestinienne. Seule une autre lecture, en décalage avec les idées reçues habituellement, permet, me semble-t-il, de donner une véritable cohérence aux événements survenus autour de la question de la bande de Gaza et pas seulement les plus récents.

\section{Les justifications israéliennes : une " guerre " peut en cacher une autre}

2 Le gouvernement israélien a, d'une voix unanime, justifié son offensive par la multiplication des tirs de roquettes revendiqués par Hamas contre les régions sud d'Israël et le refus de celui-ci de renouveler la trêve conclue en juin 2008 et arrivée à échéance le 19 décembre. L'opération «Plomb durci » ne serait ainsi que la réponse de l'« agressé », apportée au nom du "monde démocratique ", à la «terreur », en l'occurrence islamique, et au régime « obscurantiste » instauré par Hamas.

3 Les fondements de cette justification, reçue par les gouvernements et la plupart des médias non-arabes, se trouvaient pourtant ébranlés par les statistiques fournies antérieurement par l'armée israélienne elle-même : le nombre de roquettes tirées entre juillet et octobre 2008 tendait vers zéro, pour une moyenne de près de 180 par mois avant la trêve ; il dépassait à nouveau la centaine en novembre pour s'amplifier face à la crise humanitaire engendrée par le renforcement du blocus et documentée par les diverses agences des Nations-unies (Intelligence and Terrorism Information Center, The Six 
Months of the Lull Arrangement, 30 décembre 2008; UN Office for the Coordination of Humanitarian Affairs-Occupied Palestinian Territory,Humanitarian Monitor, Mensuel). La chronologie des faits permet de mettre en évidence la responsabilité israélienne dans la dégradation des conditions sécuritaires tant à Gaza qu'au sud d'Israël. Le retrait unilatéral de la bande de Gaza (et de quelques colonies du nord de la Cisjordanie) en septembre 2005, en effet, n'a pas signifié la libération de la bande de Gaza, d'ailleurs toujours considérée comme «territoire occupé » au regard des conventions internationales. A l'occupation directe s'est substituée la mise en place d'une nouvelle forme de domination beaucoup plus oppressive car extérieure. De plus et pour la première fois, à travers la gestion du passage de Rafah cette nouvelle domination jouissait du concours actif de l'Égypte et de celui, souvent oublié, de l'Union européenne. Le blocus de la bande, jamais levé en dépit des conditions de la trêve conclue à l'initiative de Hamas en juin 2008, a constitué la matière explosive des violences des derniers mois. L'opération israélienne à l'intérieur même de la bande de Gaza, qui s'est traduite le 4 novembre par la mort de 6 partisans de Hamas, a fourni le catalyseur qui devait mener à l'explosion de la fin décembre. La trêve, dont le principe restait néanmoins d'actualité pour Hamas, était devenue dans les conditions du moment quasi impossible à justifier par les responsables de Hamas auprès de sa base.

4 L'offensive contre Gaza, loin de constituer une simple réponse à la multiplication des tirs de roquettes par ailleurs bien postérieurs au lancement de ses préparatifs, n'est ainsi que le dernier avatar en date de la convergence politique observable ces dernières années aux niveaux tant international que régional -arabe, israélien et palestinien (Fatah et présidence). L'arrivée d'islamistes au pouvoir par la démocratie des urnes à l'occasion des élections législatives palestiniennes de janvier 2006, insupportable politiquement au président Abbas et aux régimes arabes réputés «modérés » comme idéologiquement aux puissances occidentales, a été utilisée par le gouvernement israélien, qui lui n'a aucune réticence à traiter avec les islamistes (comme le montre le soutien qu'il a accordé à leur développement en Palestine dans les années 1970), pour affermir et réaffirmer son refus de toute expression institutionnalisée d'une identité nationale palestinienne.

La diplomatie internationale, au-delà des condamnations convenues sur la disproportion de la «réponse » d'Israël, a de facto donné carte blanche à ce dernier pour mener ses opérations au terme qui lui convenait. Le Conseil de sécurité n'est ainsi parvenu à s'entendre (avec l'abstention des États-Unis) sur une résolution appelant -sans résultat- à « un cessez-le-feu immédiat » que le 9 janvier (785 Palestiniens, au moins, avaient déjà été tués et plus de 3300 avaient été blessés; trois civils et dix soldats israéliens avaient trouvé la mort). Silencieux depuis le début de l'offensive au nom de la doctrine rappelée le 28 décembre du « one president at a time» (un seul président à la fois), le président élu des États-Unis, Barack Obama, a accepté de parler de Gaza le 10 janvier mais pour seulement assurer vouloir mettre en place une équipe qui puisse s'engager immédiatement après son investiture dans le processus de paix au Moyen-Orient. Le 14 janvier, se refusant à prendre de quelconques sanctions contre Tel-Aviv, l'Union européenne se contentait de geler le processus de « rehaussement» de ses relations avec Israël décidé le 9 décembre à l'initiative de la présidence française ; cette décision avait sans aucun doute permis à Israël de cultiver un sentiment d'impunité alors même qu'à l'époque le Parlement européen avait décidé de reporter son vote sur la participation d'Israël aux programmes communautaires face à la poursuite du blocus de la bande de Gaza. La France, enfin, n'a pas hésité, après avoir tout fait pour en empêcher la rédaction 
au profit d'une simple déclaration, à retarder l'adoption de la résolution du Conseil de sécurité, pourtant bien peu embarrassante pour Israël comme son non-respect le montra rapidement, et pousser en avant une initiative hâtivement baptisée de «francoégyptienne » de cessez-le-feu strictement humanitaire.

Tout indique pourtant que l'offensive contre Gaza de 2008-2009 s'inscrit dans une logique israélienne déjà ancienne consistant à tout mettre en œuvre pour repousser sine die tout accord sur le fond avec les Palestiniens (plus que la question de l'État de Palestine, c'est celle de ses prérogatives, de Jérusalem et des réfugiés qu'Israël ne veut pas voir trancher). En 2005, Dov Weisglass, chef de cabinet du Premier ministre Ariel Sharon, avait ainsi reconnu que le retrait unilatéral de la bande de Gaza avait été conçu comme « la dose de formol nécessaire pour qu'il n'y ait pas de processus politique avec les Palestiniens » (Haaretz, 11 octobre 2005). En 1991, déjà, traîné par les États-Unis à la Conférence de Madrid le Premier ministre de l'époque, Itzhak Shamir, avait donné pour ordre à la délégation israélienne, dont le porte-parole était Benjamin Nétanyahou : « Négociez, avec lenteur, sans aboutir» (cité parAmnon Kapeliouk, Monde diplomatique, juillet 1996). L'offensive contre Gaza s'inscrit dans une logique comparable : imposer de facto la vision israélienne de la région et du monde à tous les acteurs politiques impliqués en exploitant l'approbation dont Israël bénéficie de la part de la communauté internationale au nom d'un engagement commun dans la «lutte contre le terrorisme». Au nom de la survie de l'État juif menacé, Israël joue exclusivement des rapports de forces à la seule fin du maintien de sa logique d'occupation militaire et de colonisation (mais également du refus de la réouverture du débat sur son identité -État juif ou État démocratique de ses citoyens ?).

7 Ainsi, en terrassant «l'infrastructure terroriste » du "gouvernement Hamas » avec le blanc-seing de la communauté internationale, c'était une nouvelle fois le nationalisme palestinien et ses institutions para-étatiques et sociales qui étaient visés sans que cette même communauté internationale ne s'en émeuve. L'observateur y verra une réplique des opérations israéliennes de 2002 qui, en mettant à bas ministères et institutions de sécurité de Cisjordanie et, déjà, de la bande de Gaza, avaient accéléré et amplifié les processus qui ont ensuite conduit la Palestine au bord de la guerre civile. En 2008, au prix certes de l'avortement violent d'un putsch ourdi par certains éléments de Fatah avec l'aide des États-Unis et d'une politique comparable à celle des régimes autoritaires de la région, Hamas, même sous blocus, était parvenu à assurer le retour d'une certaine paix civile à Gaza et avait triomphé à faire respecter tant bien que mal un véritable cessez-lefeu. C'en était sans doute trop. Hamas ne peut être qu'irresponsable. Aujourd'hui comme hier, c'est en réalité une politique de destruction du leadership palestinien, représentatif et aux affaires, du moment qui est menée, le Premier ministre Ismaïl Haniyyeh et Hamas après Yasser Arafat, Mahmoud Abbas son successeur et l'OLP (Organisation de libération de la Palestine)-Fatah.

\section{Hamas, un pion dans une partie israélienne}

Dès la fin de l'opération "Plomb durci », plusieurs hauts responsables de Hamas ou de sa mouvance (par exemple Mousa Abu Marzooq, "A decisive loss for Israel.", The Guardian, 22 janvier 2009; Mounir Chafiq, «Evaluation de la situation. », International Solidarity Movement, 27 janvier 2009) ont considéré que le cessez- 
le-feu et le retrait israéliens constituaient une confirmation de l'échec militaire et politique de l'offensive; des analystes parmi les meilleurs ont suggéré pour leur part un même constat, en apportant néanmoins quelques nuances (par exemple Bertrand Badie, " Proche-Orient : le rôle médiateur des États-Unis en question. ", chat modéré par Gaïdz Minassian, lemonde.fr, 14 janvier 2009; Gidéon Levy, "Gaza war ended in utter failure for Israel. ", Haaretz, 22 janvier 2009; Alain Gresh et Dominique Vidal dans le dossier du Monde diplomatique, février 2009). Mais une telle assertion, à distinguer de celle du renforcement des capacités de mobilisation de Hamas, dépend des objectifs fixés, l'anéantissement militaire et politique de Hamas étant alors avancé. La destruction de l'infrastructure militaire de Hamas, si tant est qu'il soit possible d'éradiquer une guérilla appuyée sur une population civile, aurait, cependant, nécessité une réoccupation totale et prolongée de la bande de Gaza. L'hypothèse d'un retour à la situation antérieure à 1994, quant à elle, avec le rétablissement d'une occupation militaire directe et pérenne dotée d'une «administration civile» israélienne en charge de la population palestinienne n'a pas vraiment été envisagée. Celle du rétablissement de Fatah, rêvé à voix haute par certains hauts responsables fathaouis, en tant que mandataire des mêmes charges, n'a pas plus été retenue ; elle aurait eu pour corollaire l'éviction de Hamas hors de l'Autorité palestinienne (à la différence de la tentative américaine de putsch de l'été 2007). De facto Hamas était donc destiné à retrouver ses responsabilités antérieures au déclenchement des hostilités face à une présidence et un cabinet Fatah de Cisjordanie toujours plus discrédités auprès de la population (Gilles Paris, «L'OLP, victime de la guerre de Gaza », Le Monde, 21 janvier 2009).

9 Victoire ou défaite, la réponse à la question dépend donc des objectifs prétendument fixés. Or les dirigeants israéliens n'ont à aucun moment fait état de buts clairs et unanimes à leur offensive. Bien plus, des informations sur de véritables divergences au sein même du cabinet et de l'armée à propos des mesures à prendre au quotidien ont franchi les barrages de l'embargo sur l'information (Aluf Benn, « Barak sought wider op, Olmert refused. », Haaretz, 22 janvier 2009, id., «.Israel's multi-faceted Gaza cease-fire. », Haaretz, 23 janvier 2009; id., «.'Viva la Gaza!'. », Haaretz, 9 janvier 2009). Divergences dans la tactique, cependant, et concurrence dans les enjeux liés aux élections de la Knesset prévues en février (avec les candidatures de Tsipi Livni, ministre des Affaires étrangères et chef du parti Kadima fondé par Ariel Sharon, face à Ehud Barak, ministre de la Défense et chef du parti travailliste), ne signifie pas nécessairement désaccord au niveau stratégique.

10 Ainsi, au delà de la réalité des destructions et des morts, après avoir été illusionnés sur l'importance et la sophistication de la menace de Hamas (la désinformation américaine sur l'Irak de Saddam Hussein a bien été oubliée) ne serions-nous pas devenus parties prenantes d'une nouvelle mise en scène israélienne, celle d'une victoire, partielle certes mais réelle, de Hamas?

Le cessez-le-feu israélien a bel et bien été une décision totalement unilatérale et strictement politique. Ni la résistance armée de Hamas (dont la faiblesse avérée renvoie à une certaine réalité des capacités militaires du mouvement mais procède aussi et surtout du concept de " guerre sans bataille » mis en œuvre par l'armée israélienne dans le cadre de sa doctrine baptisée "dahiat » et élaborée lors et à la suite de son offensive contre le Hezbollah en 2006), ni l'endurance de la population palestinienne, ni l'appel du Conseil de sécurité à un cessez-le-feu "immédiat », ni les menaces arabes de retirer l'offre de paix 
de la Ligue n'ont compté. Au sortir de l'offensive, d'ailleurs, la capacité des divers groupes militaires palestiniens (tant des mouvances islamistes, Hamas et Jihad principalement, que nationalistes, Fath, Front populaire et Front démocratique) n'a été que très partiellement et provisoirement amoindrie: les tirs de roquettes se sont poursuivis jusqu'à la proclamation palestinienne d'un cessez-le-feu conditionnel et provisoire pour une période d'une semaine et ont repris ces derniers jours faute de réouverture des points de passage entre Gaza et l'extérieur au trafic des personnes et des biens.

Il est évident pour tous les stratèges que l'opération israélienne, telle qu'elle a été menée sous sa forme et dans sa durée, ne pouvait apporter la sécurité aux régions sud d'Israël. La véritable pression israélienne, d'ailleurs, s'est faite au niveau diplomatique pour un engagement international à empêcher tout approvisionnement en armes de la bande de Gaza (et Israël a obtenu gain de cause avec la signature le 16 janvier d'un mémorandum israélo-américain sur la question et l'envoi en éclaireur d'une frégate française le 23). La logique militaire, dès lors qu'elle ne peut plus passer pour assujettie à la destruction des forces armées ennemies, relèverait plutôt de la terreur ( «Jénine à la puissance 10 » en référence à la destruction de ce camp du nord de la Cisjordanie en 2002) dans une totale déshumanisation de l'adversaire (Akiva Eldar, «Is an Israeli Jewish sense of victimization perpetuating the conflict with Palestinians ? ", Haaretz, 30 janvier 2009 à propos de Daniel Bar-Tal et Rafi Nets-Zehngut, The Israeli-Jewish Collective Memory of the Israeli-Arab/Palestinian Conflict., International Center for Cooperation and Conflict Resolution at Columbia University's Teachers College). Énoncée en termes de "dissuasion » ou de "violence disproportionnée», cette logique militaire se trouve articulée sur une politique tout aussi réelle mais cachée. Pour entretenir l'alignement international sur sa politique unilatérale en matière palestinienne, Israël mise depuis longtemps déjà sur la radicalisation de ses adversaires, radicalisation au besoin suscitée par son propre comportement. Les opposants aux politiques israéliennes, quels qu'ils soient nationalistes ou islamistes, doivent être «les ennemis de la paix » et la «modération » constitue une menace (Norman Finkelstein, «

Les dessous du dernier bain de sang à Gaza: contrer une offensive de paix palestinienne ", Info-Palestine.net, 18 janvier 2009). En tirant leurs roquettes, les Palestiniens, une nouvelle fois, auront été en « zugzwang », comme aux échecs obligés par le gouvernement israélien de jouer un coup perdant. Faute d'avoir reçu l'aval du président Bush sur la mise en œuvre de ses plans de destruction des infrastructures nucléaires iraniennes, le gouvernement israélien, en guise de «compensation » et juste avant le passage du flambeau présidentiel à Barack Obama, a pu réaffirmer sa détermination à défendre quel qu'en soit le prix sa position d'unique super-puissance régionale.

Une telle approche amène inexorablement à s'interroger sur les événements de l'été 2007 quand, le 14 juin, les forces régulières du ministère de l'Intérieur et irrégulières de Hamas (Brigades Ezzedin al-Qassam) sont passées à l'offensive et ont pris le contrôle des QG de l'ensemble des forces de sécurité liées à la Présidence et à Fatah dans la bande de Gaza. Hamas répondait ainsi, la veille de son exécution programmée, à une tentative de putsch ourdie par les États-Unis et certains éléments de Fatah contre l'Autorité palestinienne issue de la victoire de Hamas aux législatives. Les objectifs, les moyens et le calendrier de ce putsch, dont les maîtres d'œuvre étaient le général Keith Dayton, responsable au sein du Département d'État américain de la coordination en matière de sécurité avec la 
présidence palestinienne, et Muhammad Dahlan, le tout nouveau responsable du Conseil de sécurité nationale présidé par Mahmoud Abbas, avaient été révélés dans une fuite vers la presse israélienne ( Acceleration Benchmarks for Agreement on Movement and Access as Well as on the Gaza Security Situation. », Haaretz, 4 mai 2007 ; lire à ce propos, avec toutes les références bibliographiques, Jean-François Legrain, «. L'impasse politique et institutionnelle palestinienne. ", Critique Internationale (édition en ligne), n³6, juillet-septembre 2007 ; David Rose, «. The Gaza Bombshell. », Vanity Fair, avril 2008). Rien n'interdit aujourd'hui de penser qu'informés du niveau d'incompétence des forces fathaouies, les plus hauts échelons de l'establishment israélien aient décidé de précipiter par une fuite une victoire militaire de Hamas qui aurait l'«avantage » d'affaiblir encore plus le leadership de Mahmoud Abbas, de consacrer la scission géographique et politique entre la bande de Gaza et la Cisjordanie et d'obtenir le soutien unanime de la communauté internationale à une politique anti-palestinienne habillée en politique anti-terroriste. La résurrection du vieux principe du « diviser pour mieux régner ", qu'Israël avait déjà mis en œuvre dans les années 1970 pour accompagner la montée en puissance des Frères musulmans face à l'OLP, se serait cette fois-ci articulée sur l'instrumentalisation de la radicalisation, même si cela impliquait de torpiller, au moins partiellement, un plan américain auquel il était associé.

\section{La paix repoussée sine die}

Sur le fond, la politique israélienne sortirait ainsi vainqueur de l'offensive contre Gaza dès lors que «les réalités sur le terrain sont plus hostiles que jamais à un processus de paix » (Robert Malley interviewé par Philippe Grangereau, «.Reconstruire Gaza, une priorité absolue », La Libre Belgique, 26 janvier 2009). Les débats sur l'ouverture ou non du dialogue avec Hamas et sur ses conditions préalables ne sont que tergiversations de la part de la communauté internationale. Sur le fond, seule la création d'un État palestinien doté de toutes ses prérogatives aux côtés de l'État d'Israël, avec une «solution juste au droit des réfugiés " telle que l'ONU l'a traditionnellement envisagée, appuyée sur la présence d'une force internationale, pourrait amener un véritable arrêt des hostilités. Mais il s'agirait alors de faire du processus d'Oslo une parenthèse que la création d'un tel État fermerait, soit une révolution copernicienne de la diplomatie internationale.

Dès le début des années 1990, en effet, au sortir de la seconde guerre du Golfe et à la veille de l'effondrement de l'Union Soviétique, les États-Unis avaient su imposer au monde une bonne part des exigences israéliennes. Renonçant à la convocation d'une conférence internationale sous l'égide des Nations unies, organisme doté de mécanismes de contrainte, et basée sur «l'ensemble de leurs résolutions pertinentes ", la communauté internationale avait alors accepté d'apporter sa caution à de simples forums bilatéraux parrainés par les États-Unis et (nominalement) par l'Union Soviétique. Les négociations multilatérales étaient, quant à elles, consacrées aux questions qui ne touchaient en rien à la souveraineté, aux frontières et à la fin de l'état de guerre, domaines exclusivement réservés au bilatéral. En stricte continuité avec la conférence de Madrid de 1992 et les diverses négociations israélo-palestiniennes issues de l'accord d'Oslo de 1993, la « feuille de route » internationale et la conférence d'Annapolis de 2007 transforment la légalité internationale de référence (restitution des territoires occupés par la force, droit des 
réfugiés, etc.) en objet de la négociation, négociation censée une fois encore s'exercer en l'absence de tout mécanisme de contrainte, les États-Unis et leurs partenaires n'étant que de simples "facilitateurs». Loin de constituer un "proto-État», simple étape vers l'indépendance et l'État, l'Autorité intérimaire d'autonomie mise en place à partir de 1994 s'est alors transformée de facto en non-État permanent.

Un tel contexte amène à penser que seule la communauté internationale peut venir à bout du conflit par une implication massive tant politique que militaire à travers l'envoi sur place d'une force militaire d'intervention. Mais la réussite de l'envoi d'une telle force dépendra entièrement de son mandat. Une simple force d'interposition, en figeant le statu quo favorable à Israël, ne pourra qu'aller à l'échec. Qui au Conseil de sécurité, cependant, est aujourd'hui prêt à s'engager derrière une telle solution de rupture?

Le gouvernement israélien, par delà ses clivages politiciens, aurait ainsi poussé Hamas à récupérer le flambeau de la résistance nationale palestinienne d'un Fatah, absent sinon partie de l'agression menée contre la population de Gaza. Au delà des risques de condamnation d'une «disproportion" de la violence engagée et d'une réactivation de l'antisionisme et de l'antisémitisme, il aurait ainsi parié sur une redynamisation de l'alignement de la communauté internationale autour de son refus de donner un cadre étatique à l'identité nationale palestinienne au nom d'un refus commun de l'islamisme. En dépit de certaines hésitations sur l'opportunité ou non d'ouvrir un dialogue avec Hamas, la précipitation internationale, et tout particulièrement française et européenne, à participer au blocus militaire de la bande de Gaza sans faire de la réouverture de ses points d'entrée et de sortie au trafic des hommes et des biens laisse malheureusement présager que le cabinet israélien avait vu juste. Hamas et la population de Gaza, en ce cas, n'auront que, bien malgré eux, servi les intérêts de l'État d'Israël tel qu'il les conçoit aujourd'hui.

\section{RÉSUMÉS}

En décalage avec les idées reçues habituellement, l'Auteur propose une relecture de l'offensive l'armée israélienne dans la bande de Gaza (27 décembre 2008-21 janvier 2009) tant des justifications de son déclenchement avancées par le gouvernement israélien que sur l'approche de son bilan menée en termes de victoire palestinienne. Considérant qu'elle s'inscrit dans une logique israélienne consistant à tout mettre en œuvre pour repousser sine die tout accord sur le fond avec les Palestiniens, il suggère que la force réelle ou alléguée de Hamas est utilisée par le gouvernement israélien pour justifier auprès de la communauté internationale en termes de lutte contre le terrorisme une politique en réalité visant à détruire toute institutionnalisation du nationalisme palestinien.

This article is an attempt to explore new schemes for the full understandig of what was the challenge in the last war in Gaza's Strip. Between December $27^{\text {th }}$ and January $21^{\text {st }}$ nothing less than 1330 people died while 5450 others remained injuried on the Palestinian side. Ten Israeli soldiers and three civilians died in the meantime. This article is an interpretation of the Israeli way of managing the image of the Palestinian resistance movment in years, through the Muslim 
Brothers, FATHA, and now Hamas, as a danger for democracy and the western world. The figure of the trouble maker is used in order to justify a long term conflict which end Israel is the only country capable to put an end to.

INDEX

Mots-clés : Gaza, Palestine, Hamas, Israel

\section{AUTEUR}

\section{JEAN-FRANÇOIS LEGRAIN}

Jean-François Legrain (jean-francois.legrain@mom.fr) est chargé de recherches au CNRS et membre du Groupe de Recherches et d'Etudes sur la Méditerranée et le Moyen-Orient (GREMMO) - Maison de l'Orient et de la Méditerranée/Lyon. Il est l'auteur de publications dont la majorité est en ligne sur <http://www.gremmo.mom.fr/legrain> 